\title{
Testability Enhancement using Physical Design Rules in a CMOS Cell Library
}

\author{
F.C. Blom 1, J. Oliver 2, M. Rullán 2, C. Ferrer 2 \\ 1 University of Twente P.O. Box 217, NL-7500 AE Enschede, The Netherlands \\ 2 CNM (CSIC) - Univ. Autònoma de Barcelona. E 08193 Bellaterra (Barcelona), Spain
}

In order to achieve a good level of reliability we use a test strategy based on Layout Level Design For Testability (LLDFT) rules. These rules prevent the faults or reduce the appearance probability of them. We apply a practical set of LLDFT rules on the cells of the library designed on the Centre Nacional de Microelectrònica in order to obtain a highly testable cell library.

\section{INTRODUCTION}

The continuous evolution and improvement of CMOS technology provokes that certain physical failures are not accurately represented by the traditional stuck-at fault model [1].

During fabricating of an IC, disturbances in the process and contaminants (dust particles,...) may cause an incorrect behavior. The following types of defects have been reported as primary causes of CMOS IC failure: bridging, open drain or source, open gate, transmission gate opens, gate oxide shorts, parasitic transistor leakage and punch through defects.

The stuck-at fault is the dominant fault model but it is insufficient for describing faults that may occur during the fabrication of CMOS circuits. For this reason, it has been necessary to introduce realistic fault models: opens (stuck-opens, floating gates...) and shorts (stuck-ons, gate oxide shorts, bridging, floating gates...) [2] which depend on the technology and on the circuit layout. We refer as realistic faults those originated by likely physical defects induced during IC manufacturing.

So, in order to enhance the testability of the CMOS cell library designed at the Centre Nacional de Microelectrònica we have redesigned it using basic Layout-Level
Design For Testability (LLDFT) rules [3], [4] to prevent or to avoid some realistic faults.

The main advantage of applying these rules in a cell-based design process is that the new redesign for each cell has to be made only once. Otherwise, if these rules are applied in every new design, the whole circuit will need to be redesigned and this may provoke an area overhead and an increase in design time.

The short faults (including some floating gates) have a great appearance probability and are detectable by means of IDDq measurements that are assumed to be a good test technique to detect them [5].

However, we present here the main results obtained by applying basic LLDFT rules to design out some open faults and to make the remaining opens easier to be detected. Two important aspects of an IC have been measured in these simulations: electrical performance and area increase.

\section{BASIC LLDFT RULES AND RESULTS}

We have modified the layout in order to reduce the open faults appearance probability using some design rules [3], [4], [6]. The next paragraphs show the main results obtained by application of LLDFT rules on our library. These results have been 
obtained simulating each rule alone in order to know the different behavior and area increase.

The area and the electrical behavior variation (rise time, fall time, delay rise and delay fall) of the new cells are presented compared to the old results. A positive percentage corresponds to an increase in area or time with respect to the old cell, whereas a negative percentage corresponds to a better behavior than that of the old design.

\subsection{Rule a: redundancy of contacts}

Because the contacts are elements that have a high probability to produce opens.

Table I shows the results obtained over a set of cells where the contact has been duplicated.

In almost every cell, the duplication of contacts causes a equal or slightly different electrical behavior or area increase. For this reason there wouldn't be any problem to apply this rule but first duplicate important contacts (which causes hard to detect or undetectable faults if an open occurs) and after, if there is area, duplicate the other contacts.

\subsection{Rule b: partial application of a ring- shaped (based on Koeppe [7])}

An example of a ring based on Koeppe is shown in Figure 1.

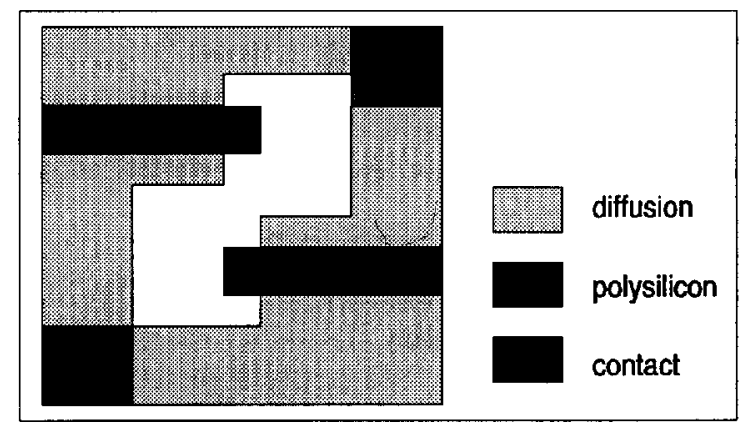

Figure 1

It is not necessary to keep the transistor width in the whole ring. Some parts of the ring are just interconnecting signal lines and may have the minimum line width of the diffusion layer (in order to reduce area and diffusion resistance).

We can see in table II that if the ring of diffusion is placed in a pull-up part, (that has a great diffusion resistance) the electrical behavior is worse than in the pull-down part (that has a low diffusion resistance). For this reason, the electrical behavior is better in a NOR gate than in a NAND gate.

Table I

Rule a: duplication of contacts

\begin{tabular}{lccccc}
\hline CELL & Area $\%$ & Rise time $\%$ & Fall time $\%$ & Delay rise \% & Delay fall \% \\
\hline INV & 0 & 0.8 & 0.8 & 0.6 & 0.6 \\
NAND & 0 & 0.5 & 0.6 & 1 & 0.7 \\
NOR & 0 & 0.2 & 0.4 & 0.2 & 0.3 \\
AND & 0 & 6.1 & 5.8 & 12 & 11.4 \\
OR & 0 & 5 & 4.2 & 4.9 & 5.7 \\
XNOR & 1 & -0.2 & 0.2 & 0.1 & -0.1 \\
AOI21 & 0 & 1.5 & 1.9 & 3.1 & 2.3 \\
AOI22 & 0 & 1.7 & 3 & 8.3 & 7.2 \\
OAI21 & 0.4 & 7 & 2.2 & 9.2 & 5.6
\end{tabular}


Table II

Rule b: partial application of a ring shaped

\begin{tabular}{lccccc}
\hline CELL & Area $\%$ & Rise time $\%$ & Fall time \% & Delay rise \% & Delay fall \% \\
\hline NAND & 6.4 & 44 & -8.7 & 36.7 & -6.2 \\
NOR & 5.6 & 0.8 & 1.8 & 0.2 & -0.3 \\
AND & 3.1 & 4.8 & 8.8 & 14.7 & 15.8 \\
OR & 15 & 4.6 & 10.5 & 7.4 & 8 \\
OAI21 & 2 & 7.1 & 2.8 & 5.1 & 4.5 \\
OAI22 & 10 & 1.3 & 1.3 & 3.6 & 4.8 \\
\hline
\end{tabular}

2.3. Rule c: duplication of interconnection layers and inputs

The duplication of lines causes in general, an enormous area increase and worse electrical behavior (see table III) except for the NOR gate. This kind of rule could only be applied when an equilibrium between the added cost (in area or electrical behavior) and the improvement in the faults occurrence is important.

\subsection{Rule d: placing according to the} signal flow

In this case the purpose is to prevent feedback bridging [4] within a cell. This causes a very poor electrical behavior in the AND gate (see table IV), but a good electrical behavior in the OR gate. This rule is strongly layout dependent and it is difficult to establish an optimal criterion to use it.

Table III

Rule c: duplication of inputs

\begin{tabular}{lccccc}
\hline CELL & Area $\%$ & Rise time \% & Fall time \% & Delay rise \% & Delay fall \% \\
\hline INV & 25 & 4 & 4 & 7 & 7 \\
NAND & 31 & 3.7 & 4.5 & 5.3 & 4.3 \\
NOR & 0 & 1.5 & 0.6 & 1.9 & 0.8 \\
OR & 36.8 & 1.3 & 2.3 & 1.5 & -0.1 \\
AND & 25.6 & 10 & 10 & 26 & 21 \\
\hline
\end{tabular}

Table IV

Rule d: placing according to the signal flow

\begin{tabular}{lccccc}
\hline CELL & Area $\%$ & Rise time $\%$ & Fall time $\%$ & Delay rise \% & Delay fall \% \\
\hline AND & 0 & 9.6 & 7.9 & 8.5 & 6.1 \\
OR & 0 & 5.6 & 4.9 & 4.2 & 4.6 \\
\hline
\end{tabular}


In Figure 2 we can see a NOR cell and a redesigned cell. We have applied rule $b$ in the pull-down path. Again, it is not necessary to keep the transistor width in the whole ring. If an open occurs in the common contact it could be detected as a stuck-at fault, and for this reason we have only multiplied the rest of them (rule a). On the other hand we have also duplicated the input gate to avoid an open in this layer (rule c).

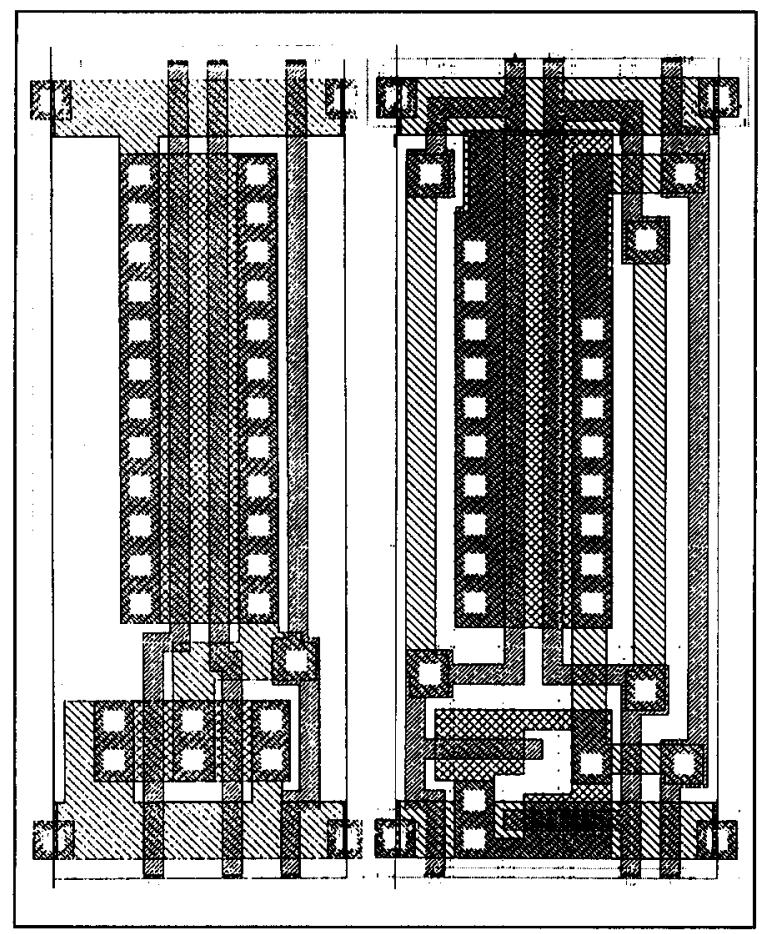

Figure 2: old and redesigned NOR cell

\section{CONCLUSIONS}

In short, the utilization of these rules seems reasonable in order to avoid some opens or to use easier fault models (i.e. classical stuck-at), when the new cells do not exceed the $10 \%$ of variation from the nominal value of the standard cells.

The application of these rules implies that the area overhead is always under the $10 \%$, except when the layers and inputs (rule c) have been duplicated. The electrical behavior is degraded around the $10 \%$ except when rule $b$ (application of the Koeppe ring) has been applied in some cells.

Furthermore, these results show that it is possible to improve the circuit testability by improving the physical design. Although our results have been focused on open faults avoidance and this work could be applied also on bridging faults.

Now, our future work is to extend this methodology to more complex cells and also to the routing area applying this methodology to ISCAS benchmark circuits. We also want to make the detection of some faults (shorts, floating gate,...) easier by IDDq measurements, introducing built-in current sensors in a cell-based design strategy.

\section{REFERENCES}

1. R.L. Wadsack, Fault Modeling and Logic Simulation of CMOS and NMOS Integrated Circuits, Bell Syst., Tech. Journal, vol. 57, n², pp. 1449-74, 1978.

2. W. Maly; Realistic Fault Modeling for VLSI Testing; Proc. 24 Design Automation Conf. (DAC), pp. 173-180, 1987.

3. J.P. Teixeira, F.M. Gonçalves, J.J.T. Sousa; Layout-driven testability enhancement; European Test Conference, pp. 101-109, 1991.

4. H.T. Vierhaus; Rule-based design for testability- The extest approach; Proc. Compeuro, pp. 949-952, 1987.

5. V.H. Champac, R. Rodríguez, J.A. Segura, J. Figueras y J.A. Rubio; "Fault modeling of Gate Oxide Shorts, Floating Gate and Bridging Failures in CMOS Circuits; European Test Conference, pp. 143148, 1991.

6. F.C. Blom, J. Oliver, M. Rullán, C. Ferrer; Layout-Level Design for Testability Strategy Applied to a CMOS Cell Library; Workshop Design for Testability; Venice, 1993 (to be published).

7. S. Koeppe; Optimal layout to avoid CMOS stuck-open faults; 24th ACM/IEEE Design Automation Conference, pp.829-835, 1987. 